\title{
Article
}

\section{Discrimination of human stem cells by photothermal microspectroscopy}

Grude, Olaug, Nakamura, Takahiro, Hammiche, Azzedine, Bentley, Adam J., Martin, Francis L, Pollock, Hubert M., Kinoshita, Shigeru and Fullwood, Nigel J.

Available at http://clok.uclan.ac.uk/16311/

Grude, Olaug, Nakamura, Takahiro, Hammiche, Azzedine, Bentley, Adam J., Martin, Francis L ORCID: 0000-0001-8562-4944, Pollock, Hubert M., Kinoshita, Shigeru and Fullwood, Nigel J. (2009) Discrimination of human stem cells by photothermal microspectroscopy. Vibrational Spectroscopy, 49 (1). pp. 22-27. ISSN 0924-2031

It is advisable to refer to the publisher's version if you intend to cite from the work. http://dx.doi.org/10.1016/j.vibspec.2008.04.008

For more information about UCLan's research in this area go to http://www.uclan.ac.uk/researchgroups/ and search for <name of research Group>.

For information about Research generally at UCLan please go to http://www.uclan.ac.uk/research/

All outputs in CLoK are protected by Intellectual Property Rights law, including Copyright law. Copyright, IPR and Moral Rights for the works on this site are retained by the individual authors and/or other copyright owners. Terms and conditions for use of this material are defined in the policies page. 


\section{DISCRIMINATION OF HUMAN STEM CELLS BY PHOTOTHERMAL MICROSPECTROSCOPY}

Olaug Grude ${ }^{1,2 \star}$, Takahiro Nakamura ${ }^{3}$, Azzedine Hammiche ${ }^{2}$, Adam J. Bentley ${ }^{1}$, Francis L. Martin ${ }^{1}$, Hubert M. Pollock ${ }^{2}$, Shigeru Kinoshita ${ }^{3}$, Nigel J. Fullwood ${ }^{1}$

Vibrational Spectroscopy 49, 22-27 (2009)

1. Biomedical Sciences Unit, Department of Biological Sciences, Lancaster University, Lancaster, UK

2. Spectroscopy Group, Department of Physics, Lancaster University, Lancaster, UK.

3. Department of Ophthalmology, Kyoto Prefectural University of Medicine, Kyoto, Japan

* corresponding author contact details: o.grude@lancaster.ac.uk.

Phone +44 (0) 1524593 474. Fax +44 (0) 1524853854

\section{ABSTRACT}

Stem cells have great potential in clinical medicine. Sensitive methods for stem cell identification are a requirement for the development of medical interventions involving these cells. To date, a definitive stem cell marker has not been discovered. We are exploring the use of photothermal microspectroscopy (PTMS) for the purpose of stem cell characterisation and identification in human corneal epithelium. PTMS measures heat fluctuations associated with infrared radiation absorption. The technique is advantageous over existing Fourier transform infrared (FTIR) spectroscopy methods in having a spatial resolution which is not diffraction limited, thus allowing examination at a subcellular scale. PTMS measurements are unaffected by IR opacity of the sample, giving the method a further edge in comparison to FTIR spectroscopy. We show that PTMS spectra can be used for the characterisation of stem cells and differentiated cells in the human corneal stem cell model. We demonstrate for the first time that PTMS spectra derived from these cell types segregate into separate data clusters after principal component analysis. The predominant wavenumbers responsible for this separation appear to be associated with nucleic acid structure and function. PTMS offers great promise as a technique for stem cell identification in tissue samples where spatial resolution at the cellular scale or better is required.

KEY WORDS: photothermal microspectroscopy, stem cells, human, principal component analysis

\section{INTRODUCTION}

Stem cells (SCs) are the origin of all adult cell types, including themselves. Adult tissues with continuous cell turnover, such as the blood, epidermis and cornea contain tissue-specific SC populations. Adult SCs are slow-cycling, have a high capacity for self-renewal and a life cycle as long as the lifetime of the parent organism ${ }^{1}$. SCs divide either symmetrically to produce two identical daughter SCs, or asymmetrically to give rise to one SC and one transit amplifying cell. This leads to the generation of all differentiated cell types ${ }^{2}$.

The corneal epithelium is a well-understood SC system. Cell populations within this system are segregated and spatially well-defined. The model therefore lends itself attractively to the study of SCs and differentiated cell types within their native tissue locations. SCs are usually confined to their "niche", where the cells are maintained and protected ${ }^{3}$. The corneoscleral limbus is acknowledged as the location for the corneal epithelial SC niche ${ }^{4,5}$. Light micrographs of the intact human corneoscleral limbus and cornea are shown in figures 1 and 2, respectively. Limbal trauma or disease resulting in corneal stem cell deficiency are associated with severe loss of function of the ocular surface. Transplantation of autologous limbal SCs in patients with unilateral limbal SC deficiency can however result in complete restoration of the compromised cornea ${ }^{6}$. 


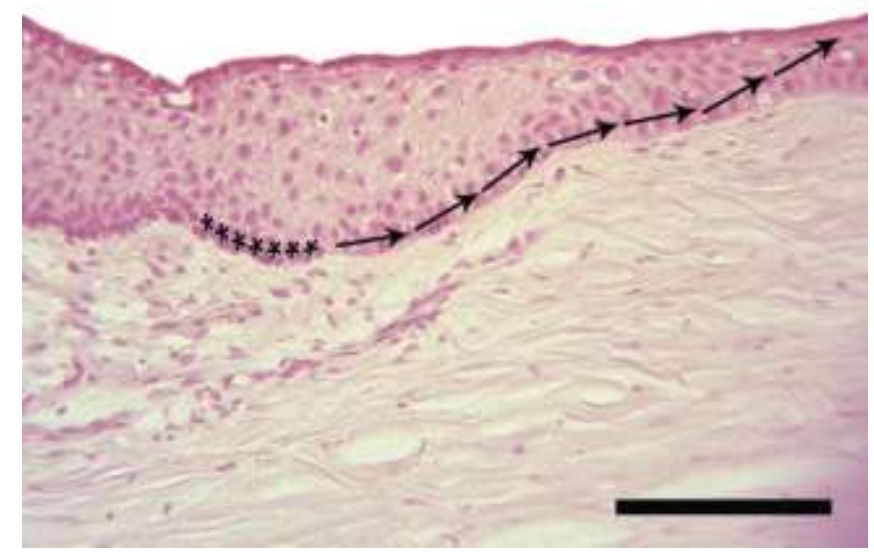

FIGURE 1 Light micrograph of the corneoscleral limbus in human cornea. The top cell layer (darker in colour) is the epithelium. The lower cell layer is the corneal stroma. The asterisks represent the location of the stem cell niche in the basal cell layer of the limbal epithelium. The arrows illustrate the migration pathway of the epithelial cells as they leave the stem cell niche and differentiate. Scale bar is equal to $100 \mu \mathrm{m}$.

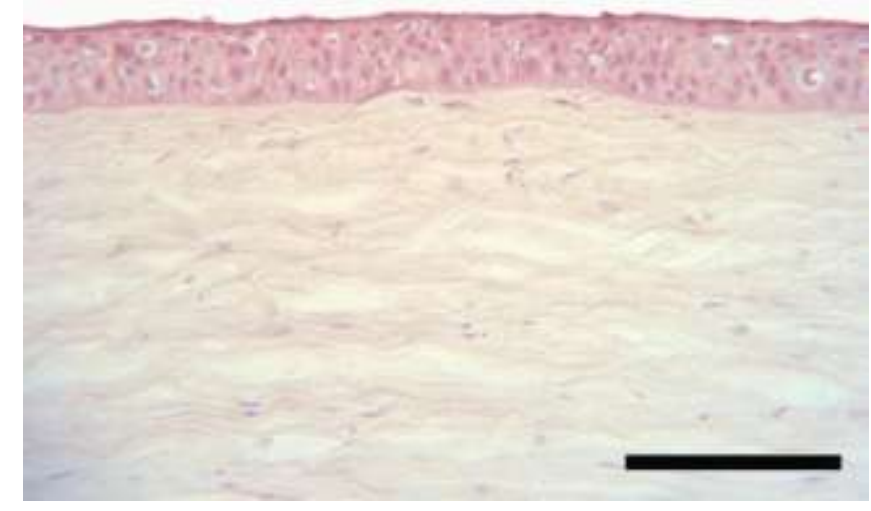

FIGURE 2 Light micrograph of human cornea. The top cell layer (darker in colour) is the epithelium. The lower cell layer is the corneal stroma. Scale bar is equal to $100 \mu \mathrm{m}$.

The higher aim of finding a universal SC marker goes hand-in-hand with the exploration of SCs in their environment. Any method which can be applied to aid the elucidation of SC properties will expand our understanding of how SCs function. Highly spatially resolved Fourier Transform Infrared (FTIR) spectroscopy in combination with powerful statistical analysis techniques have been proven valuable in this respect. FTIR spectroscopy is based on the principle that tissues absorb infrared (IR) wavelengths corresponding to the resonant frequencies of the vibrational bonds within their structure ${ }^{7}$. The IR spectrum of a tissue sample will consist of a combination of absorption peaks which will tend to be unique to the type of tissue examined. The technique has been applied for the characterisation and subsequent discrimination of bovine and human corneal SCs and differentiated cells in tissue 
sections $^{8,9}$. On the basis of FTIR spectroscopy image mapping, specific infrared spectral features have been proposed as markers for SCs in the gut ${ }^{10}$. FTIR spectroscopy has also been used successfully for the detection of subtle intracellular changes associated with Alzheimer's disease ${ }^{11}$, bone and cartilage disorders ${ }^{12}$ and different stages of the cell cycle ${ }^{13}$. The technique has furthermore been proven extremely sensitive in the diagnosis and grading of pre-malignant and malignant neoplasia ${ }^{14-16}$

Photothermal microspectroscopy (PTMS) is a technique which in effect combines all the benefits of FTIR spectroscopy ${ }^{17}$ with the advantages of non-optical sensors. The PTMS experimental set-up consists of a bench-top FTIR spectrometer interfaced with an atomic force microscope which is fitted with a near-field resistive thermal sensing probe. The technique is used for detection of IR absorbance associated heat release. The probe itself detects this temperature change as a function of voltage change ${ }^{18}$. Figure 3 shows a schematic illustration of the PTMS set-up.

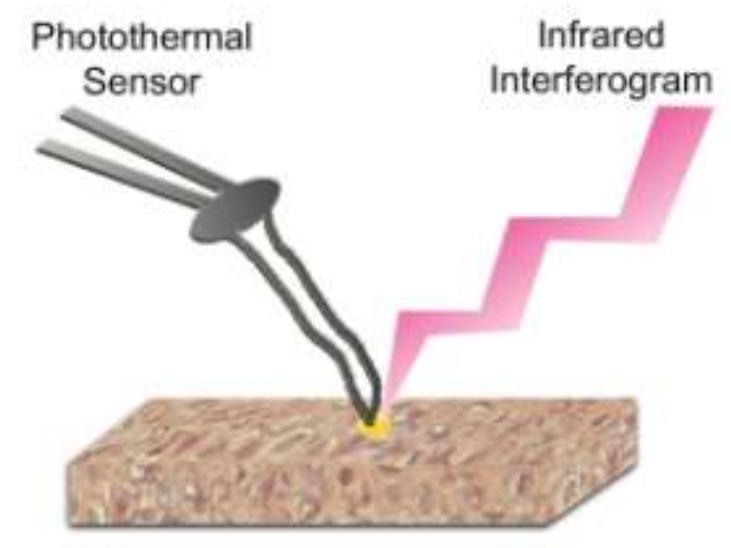

FIGURE 3 schematic illustration of the principles involved in photothermal microspectroscopy (PTMS). Midinfrared radiation is generated by a blackbody radiator and modulated by a Michelson's interferometer contained within a Fourier transform infrared (FTIR) spectrometer. This radiation is focused onto the biological tissue section via a custom-built optical interface. IR absorption-associated heat release at the IR focal point (in yellow) is detected with a photothermal sensor.

The absence of optical components in the PTMS sensor means that the technique circumvents the diffraction-limited spatial resolution that conventional FTIR spectroscopy techniques are governed by. With conventional FTIR spectroscopy, the practical spatial resolution will be around $12 \mu \mathrm{m}$ when using highly brilliant Synchrotron radiation only available at electron accelerator rings. With a benchtop radiation source, the best resolution which does not compromise good signal-to-noise characteristics of the acquired spectra will be in the region of $50 \mu \mathrm{m}^{19}$.

The spatial resolution with PTMS is limited mainly by the dimensions of the probe tip. A spatial resolution as small as $2 \mu \mathrm{m}$ is obtainable with a bench-top IR radiation source and the Wollaston probes used in the experimental set-up ${ }^{20}$. Human corneal epithelial cells are typically 30-50 $\mu \mathrm{m}$ in diameter and with nuclei of approximately 5-10 $\mu \mathrm{m}$ across ${ }^{21}$. PTMS is therefore well-suited for singlecell interrogation, even single-organelle examination under optimum conditions.

In this paper, we describe the use of PTMS for characterisation and discrimination of SCs and differentiated cells in tissue sections of human corneal epithelium. 


\section{METHODS}

\section{Sample preparation}

Corneal specimens were obtained from the North-West Lions Eye Bank, Seattle, WA. The corneas were from male Caucasians. Eyes were harvested within $12 \mathrm{~h}$ of death, the corneas placed in Optisol corneal preservation medium (Chiron Vision, CA) and stored at $4^{\circ} \mathrm{C}$ for up to 5 days before use. Corneal samples were dissected and frozen. Cryosections (10 $\mu \mathrm{m}$ thick) were collected onto $\mathrm{BaF}_{2}$ slides (Photox Optical Systems, Sheffield, UK). The sections were stored in desiccators until required. Parallel sections were stained with haematoxylin and eosin for histological comparison.

\section{PTMS instrumentation}

Our PTMS system consisted of an optical interface (Specac, Orpington, UK) in the chamber compartment of the FTIR spectrometer (Vector 22 model, Bruker Optics, Coventry, UK). The scanning probe microscope (Explorer model, Veeco Instruments, Santa Barbara, CA, USA) was equipped with a Wollaston wire resistive thermal probe (Veeco Instruments). The probe tip and sample were brought to the IR focal point to maximise the photothermal signal.

\section{Data acquisition and processing}

Prior to sample measurements, probe tips were cleaned by Joule heating by applying a current of 100 $\mathrm{mA}\left(\sim 600^{\circ} \mathrm{C}\right)$. Photothermal interferograms were averaged over time (typically 3,000 co-additions) for enhancement of signal-to-noise characteristics, followed by conversion by Fourier transformation to spectra displaying photothermal amplitude expressed as a function of wavenumber $\left(\mathrm{cm}^{-1}\right)$. Spectral data were sampled at $8 \mathrm{~cm}^{-1}$ intervals. Atmospheric background spectra were taken in non-contact mode and inspected to ensure that the tip was free from biological contamination. Sample spectra were taken with the probe lowered onto the cell populations of interest, which were the stem cells $(n=26)$ in the basal limbal region and differentiated cells $(n=26)$ from the mid-periphery of the corneal epithelium.

\section{Data processing and statistical analysis}

Data acquisition and atmospheric background correction were carried out in OPUS software (Bruker Optics). Baseline correction, normalisation and principal component analysis (PCA) were performed with Pirouette software (Infometrix Inc., Bothell, WA, USA). Narrowband regions containing noise arising from mains electric pick-up were excluded from analysis. These regions were centered on $1082 \mathrm{~cm}^{-1}$ and on $1442 \mathrm{~cm}^{-1}$, corresponding to the $2^{\text {nd }}$ and $3^{\text {rd }}$ harmonics from the mains electric vibrations respectively. Spectral regions outside of the biomolecular range from $1800 \mathrm{~cm}^{-1}$ to $900 \mathrm{~cm}^{-1}$ were also excluded. Median spectra were calculated in Microsoft Excel.

Mann-Whitney calculations were performed in R statistical software 2.2.1. The Mann-Whitney test is non-parametric and calculates the probability of two groups of values being drawn from the same distribution by chance. A calculated $P$ value of 0.01 means that there is only a $1 \%$ probability of the two groups of values being drawn from the same distribution. In other words, $99 \%$ confidence that the two groups are statistically different.

Linear discriminant analysis (LDA) computations of class-specific loadings were also calculated in $\mathrm{R}$ from the factor-specific loadings found by $\mathrm{PCA}^{22}$. The class-specific loadings display the variance of each cell class relative to the all classes in the data set. In the case of a data set with only two classes, the two class-specific loading plots will therefore be mirror-images of each other. The absolute values of these two loading plots will in this case be the same. Displaying the absolute loading plot will avoid artifacts of zero loading value between successive data points with opposite signs.

Quadratic discriminant distributions were calculated from PCA factors in the Discriminant Analysis add-on toolbox for Matlab ${ }^{23}$. 
RESULTS:

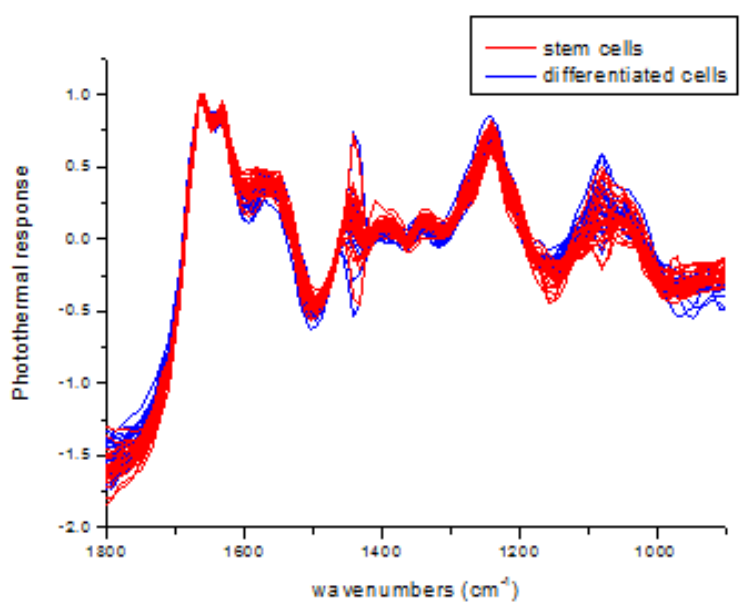

FIGURE 4 All acquired spectra, showing subtle but consistent variations with cell type. Large portions of spectral similarities across cell type arise from all cells being part of the same tissue type. Narrowband fluctuations centred on $1082 \mathrm{~cm}^{-1}$ and on $1442 \mathrm{~cm}^{-1}$ correspond to pick-up noise from the $2^{\text {nd }}$ and $3^{\text {rd }}$ harmonics from the mains electric vibrations. These two narrowband regions were therefore excluded from statistical analysis.

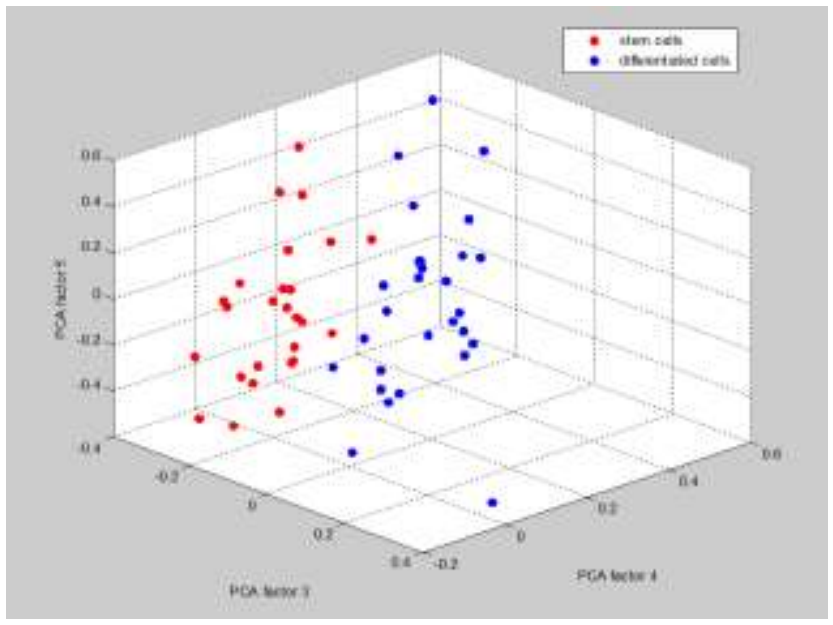

FIGURE 5 Principal component analysis cluster plot showing perfect segregation of data derived from PTMS measurements of stem cells and differentiated cells of human corneal epithelium. 


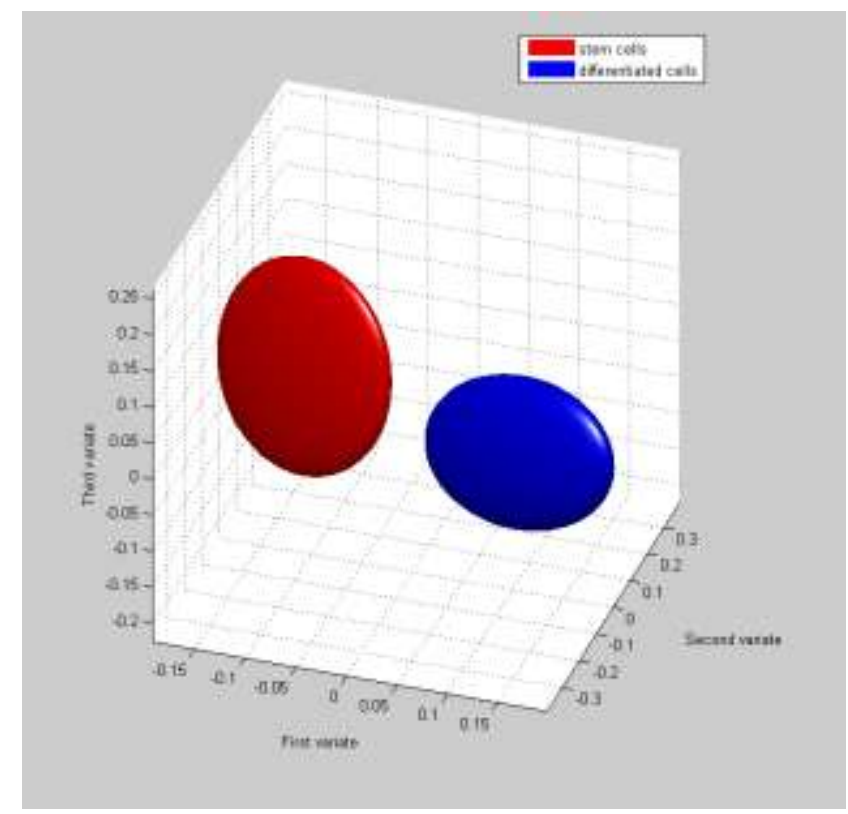

FIGURE 6 Quadratic discriminant analysis representation of principal component data points shown in figure 5 , demonstrating the Gaussian distributions of the data clusters from the different cell populations of stem cells and differentiated cells.

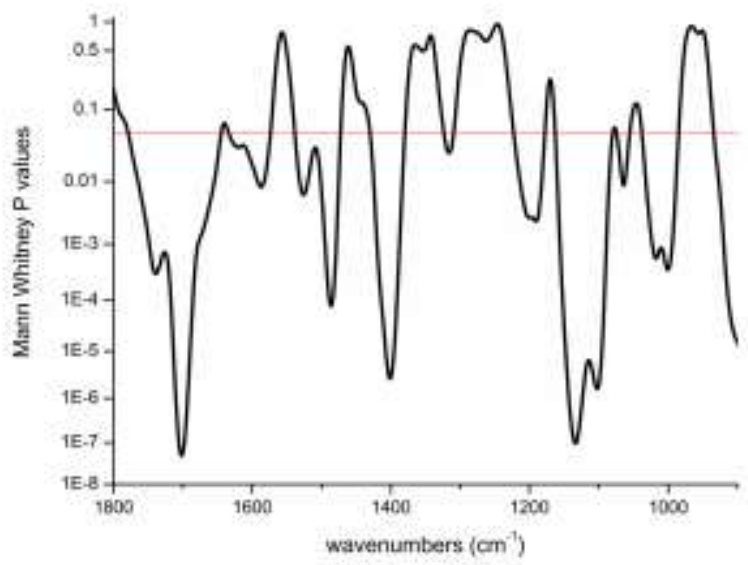

FIGURE $7 \mathrm{P}$ values from the Mann-Whitney test for statistically significant differences between spectra from stem cells and differentiated cells, shown as a function of wavenumber. The red line represents the $95 \%$ confidence level. All portions of the plot below this line are statistically significant to this level of confidence or better. 


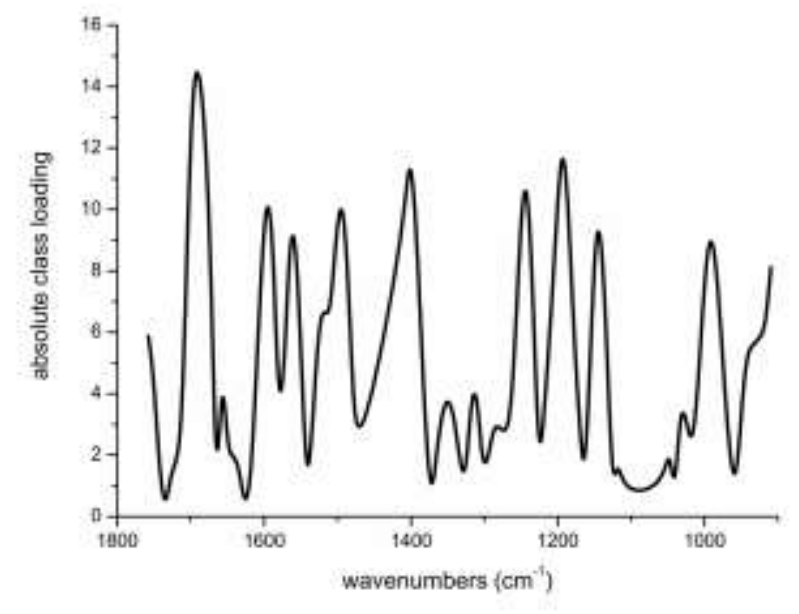

FIGURE 8 Absolute values of class-specific loadings. These were calculated by linear discriminant analysis from factor-specific loadings computed by principal component analysis. The peaks represent the principal wavenumbers responsible for the separation of cell classes in the principal component clusterplot (see figure 5).

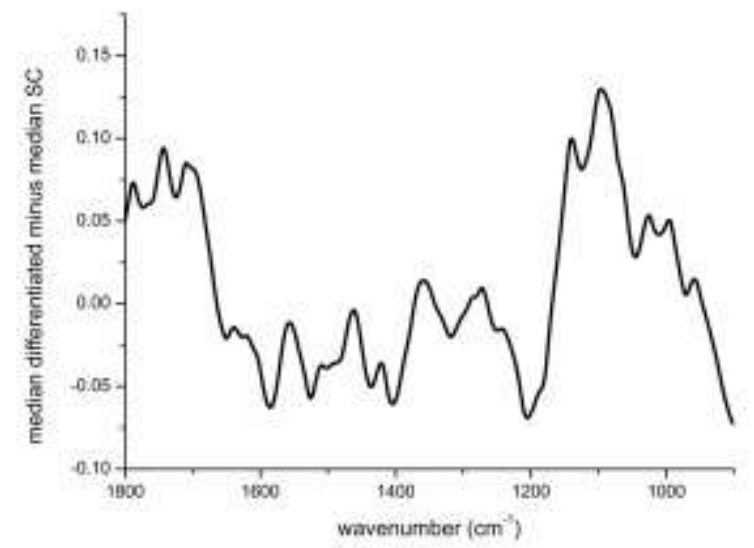

FIGURE 9 Difference spectrum of median differentiated cell spectrum minus median stem cell spectrum. Troughs and peaks highlight the main wavenumbers at which photothermal spectra from the two cell types differ.

Figure 4 shows all acquired spectra from both classes of cells. Figure 5 demonstrates segregation of data derived from SCs and differentiated cells into separate clusters after PCA. Figure 6 shows the quadratic discriminant representations of the same data. Each disc has the shape of the Gaussian distribution of the data cluster used for its generation.

Figures 7 to 9 highlight the wavenumbers at which spectra from SCs and differentiated cells are the most dissimilar. Figure 7 shows the $P$ values from the Mann-Whitney test for statistical significant differences between spectra from the two cell types. The most highly significant values are those with lowest $P$ values. The red horizontal line describes the $95 \%$ confidence level, and all portions of the graph below this line are statistically significant above this level of confidence. Figure 8 shows the absolute values of the class-specific loadings from PCA. This plot identifies the principal 
wavenumbers responsible for the separation of spectral data into segregated clusters ${ }^{16}$. Figure 9 shows the subtraction spectrum of the median SC spectrum from the median differentiated cell spectrum.

The main wavenumbers responsible for the separation of spectra from SCs and differentiated cell types are those in the region of $1710-1700 \mathrm{~cm}^{-1}$ (figures 6,7 and 8), around of $1590 \mathrm{~cm}^{-1}$ (figures 7 and 8), the region of $1480 \mathrm{~cm}^{-1}$ (figure 7 and 8), the region of $1400 \mathrm{~cm}^{-1}$ (figures 7,8 and 9), $1240 \mathrm{~cm}^{-1}$ (figure 8), $1200-1190 \mathrm{~cm}^{-1}$ (figures 7,8 and 9), region $1155 \mathrm{~cm}^{-1}$ (figures 7,8 and 9) and the region around $1000-950 \mathrm{~cm}^{-1}$ (figures 7,8 and 9 ).

Differences in the region of $1710-1700 \mathrm{~cm}^{-1}$ may be attributed to $\mathrm{C}=\mathrm{O}$ stretching vibrations of nucleic acids ${ }^{7}$. Activity around $1590 \mathrm{~cm}^{-1}$ is most certainly due to $\mathrm{C}=\mathrm{N}$ stretching in guanine. The vibrational bond responsible for the $1480 \mathrm{~cm}^{-1}$ peak can be more difficult to assign. The peak is in the middle of the protein range from around $1640 \mathrm{~cm}^{-1}$ to $1240 \mathrm{to}^{-1}{ }^{-124}$, and also at one extreme of the end of the complex absorption profile from $1480 \mathrm{~cm}^{-1}$ to $1450 \mathrm{~cm}^{-1}$ arising from $\mathrm{CH}_{2}$ scissoring and $\mathrm{CH}_{3}$ asymmetric bending vibrations of lipids, proteins and nucleic acids ${ }^{7}$. Absorptions at $1400 \mathrm{~cm}^{-1}$ can be due to $\mathrm{COO}^{-}$symmetrical stretching vibrations of fatty acids and amino acids ${ }^{7}$ or $\mathrm{C}-\mathrm{H}$ bend in amino acid residues ${ }^{24} .1240 \mathrm{~cm}^{-1}$ is the peak for $\mathrm{C}-\mathrm{N}$ stretch and $\mathrm{N}-\mathrm{H}$ bend of the protein amide III. The region around $1200-1190 \mathrm{~cm}^{-1}$ is within the broadband phosphate range from $1200-900 \mathrm{~cm}^{-1} 25$ and may therefore be assigned to phosphate activity. The narrow region around $1160-1150 \mathrm{~cm}^{-1}$ is associated with carbohydrate ${ }^{16}$ or with C-O stretch in DNA ${ }^{24}$. Finally, the band around $1000-950 \mathrm{~cm}^{-1}$ is associated with sugar ring vibrations ${ }^{26}$ and $\mathrm{C}-\mathrm{O}$ and $\mathrm{C}-\mathrm{C}$ stretch in DNA ${ }^{24}$.

\section{DISCUSSION}

Covalently bonded atoms absorb IR radiation corresponding to the specific resonant frequencies of their vibrational motions ${ }^{24}$. It is on this basis that FTIR spectroscopy can be used for characterisation of biological materials such as cultured cells or tissue sections. PTMS is a technique which combines the benefits of conventional FTIR spectroscopy with the advantages of near-field non-optical sensors. PTMS overcomes the diffraction limitations which restrict the spatial resolution of beam-focussing sensors ${ }^{27}$, like those employed with FTIR spectroscopy, to a practical minimum of around $12 \mu \mathrm{m}$ in the IR range ${ }^{19}$. This optimum level of resolution requires the use of synchrotron radiation, which is only available at very few electron accelerator rings worldwide. The spatial resolution of PTMS is limited mainly by the dimensions of the probe tip and by the thermal diffusion length in the sample material $\left.\right|^{28}$. In practical terms, this allows for spatial resolution in the region of $2 \mu \mathrm{m}$ even when using a bench-top radiation source. Single cell measurements are therefore perfectly possible with this instrumentation. Furthermore, PTMS circumvents the inherent shortcoming with FTIR detectors of non-linearity at high absorbance values, which causes distortions of relative signal intensities and band shapes ${ }^{29}$. PTMS is based on the detection of IR absorption associated heat release. IR opacity of the sample does therefore not cause signal distortion. This makes PTMS advantageous over conventional FTIR spectroscopy in samples where IR transmission can be low, such as cellular material containing nuclei with densely packed chromatin strands ${ }^{30}$.

IR spectroscopy data from different cell populations within the same tissue will demonstrate a lot of similarities as well as some variation between the cell types. Powerful statistical analysis techniques are often required to extract consistent but subtle variance between cell types. For this purpose PCA, being a form of multivariate analysis, is a suitably sensitive statistical analysis method. PCA reduces the number of data entries per spectrum with emphasis on variance rather than similarities in the data set as a whole, with the end result of describing the data in typically 5 to 9 principal components (PCs) or dimensions. Of these, three PCs can be displayed at any one time in a pseudo-3D cluster plot. Each PC contains a certain percentage of variability of the original data, and each cluster plot will display the extent of variation of all spectra in the three selected dimensions. Closeness of data points in multivariate hyperspace implies similarity, whereas relative separation means more differences ${ }^{32}$. 
Medical interventions involving the use of SCs offer great clinical potential ${ }^{3,32}$. To date, many tissuespecific adult SC populations have not been identified, and a definitive stem cell marker universal to all tissue types has not been found. The absence of idiosyncratic morphology or other features specific to SCs can make their presence difficult to pinpoint ${ }^{32}$. That a definitive SC "marker", universal to all tissue types, is yet to be identified is perhaps more of a reflection of the relative featurelessness of the cell type than a shortcoming of the decades of scientific effort invested in this quest. The search for markers specific to the limbal SC population has lead to the discovery of a number of molecules selectively expressed by these cells and not by other cell populations in the same tissue, including p63 and the keratin pair $\mathrm{K} 5 / \mathrm{K} 14$. The absence of gap junction proteins is a further limbal SC indicator ${ }^{34}$.

IR spectroscopy methods are used for examination of all biomolecules present in a sample. It can therefore be advantageous over methods used to identify cells on the basis of single molecular types only. Previous work ${ }^{35}$ has demonstrated that bench-top PTMS is more sensitive than synchrotron radiation FTIR spectroscopy in terms of ability to discriminate between SCs, transit amplifying and terminally differentiated cells in tissue sections of bovine cornea. Proposed reasons for this finding were two-fold. Firstly, the spatial resolution with PTMS is at least as good as that obtained with synchrotron radiation FTIR spectroscopy, thus reducing the chance of sampling across cell population boundaries. Secondly, PTMS measures IR absorption directly rather than, as with FTIR spectroscopy, as a computation from infrared transmission through a sample.

We have shown that PTMS in conjunction with PCA can separate spectra derived from SCs and differentiated cells into discrete data clusters in multidimensional hyperspace. The predominant wavenumbers responsible for the segregation are found around the $1700 \mathrm{~cm}^{-1}$ peak indicative of nucleic acids ${ }^{7}$ and in the range $1490 \mathrm{~cm}^{-1}$ to $1000 \mathrm{~cm}^{-1}$ which indicates IR absorption by nucleic acids and/or changes in chromatin structure ${ }^{36}$. Further differences in the region from $1000-950 \mathrm{~cm}^{-1}$ denoting $\mathrm{DNA}^{23}$ reinforces the inference that the spectral dissimilarities between the SC and differentiated cell populations are extracted from changes in nucleic acid composition and structure. The PTMS spectral resolution of $8 \mathrm{~cm}^{-1}$ does not allow for a higher degree of specificity as regards assignment of vibrational bond peaks. Statistical analysis of PTMS spectra from SCs and differentiated cells in sections of human cornea supports previously published FTIR spectroscopy results on the same model in humans ${ }^{8}$ and PTMS work on the same tissue type from bovine ${ }^{35}$. The finding that PTMS detects differences in nucleic acids between the SC and differentiated cell populations in the human corneal epithelium is consistent with reports of SC differentiation being triggered by chromatin modifications ${ }^{37}$.

\section{CONCLUSION}

We have shown that PTMS can be used for characterisation of SCs and differentiated cells in tissue sections of human cornea. We demonstrate for the first time that PTMS spectra collected from these two cell types form distinctly separate data clusters after PCA. Some of the spectral bands responsible for the separation of data derived from SCs and differentiated cells correspond to nucleic acid structure and function. Each PTMS spectrum provides a broad range of biomolecular information, in contrast to techniques which identify cells on the basis of the presence or absence of single molecular groups. The work presented in this article is promising with respect to the development of PTMS spectra as sensitive markers for SC in tissue samples where high spatial resolution is a requirement.

\section{ACKNOWLEDGEMENTS}

This work was supported by the Biotechnology and Biological Sciences Research Council, UK (grant number BB/D010055/1) and the Engineering and Physical Sciences Research Council, UK. 
[1] P.A. Hall, F.M. Watt, Development 106 (1989) 619.

[2] N.D. Theise, R. Harris, in Handbook of Experimental Pharmacology vol. 174, SpringerVerlag Berlin Heidelberg, 2006, p. 389.

[3] E. Fuchs, J.A. Segre, Cell. 100 (2000) 143.

[4] G. Cotsarelis, S.Z. Cheng, G. Dong G, T.T. Sun, R.M. Lavker, Cell 21 (1989) 201.

[5] H.S. Dua, V.A. Shanmuganathan, A.O. Powell-Richards, P.J. Tighe, A. Joseph, Br. J. Ophthalmol. 89 (2005) 529.

[6] H.S. Dua, A. Azuara-Blanco, Br. J. Ophthalmol. 84 (2000) 273.

[7] M. Jackson, M. Mantsch, Biomolecules, Wiley-Liss (1996) 311.

[8] A.J. Bentley, T. Nakamura, A. Hammiche, H.M. Pollock, F.L. Martin, S. Kinoshita, N.J. Fullwood, Mol. Vis. 13 (2007) 237.

[9] M.J. German, H.M. Pollock, B. Zhao, M.J. Tobin, A. Hammiche, A.J. Bentley, L.J. Cooper, F.L. Martin, N.J. Fullwood, Invest. Ophthalmol. Vis. Sci. 47 (2006) 2417.

[10] M.J. Walsh, T.G. Fellous, A. Hammiche, W.R. Lin, N.J. Fullwood, O. Grude, F. Bahrami, J.M. Nicholson, M. Cotte, J. Susini, H.M. Pollock, M. Brittan, P.L. Martin-Hirsch, M.R. Alison, F.L. Martin, Stem Cells 26 ( 2008) 108.

[11] L.M. Miller, Q. Wang, T.P. Telivala, R.J. Smith, A. Lanzirotti, J. Miklossy, J. Struct. Biol. 155 (2006) 30.

[12] A. Boskey, N. Pleshko Camacho, Biomaterials 28 (2007) 2465.

[13] S. Boydston-White, M. Romeo , T. Chernenko , A. Regina, M. Miljković , M. Diem, Biochim Biophys Acta 7 (2006) 908.

[14] S. Mordechai, R.K. Sahu, Z. Hammody, S. Mark, K. Kantarovich, H. Guterman, A. Podshyvalov, J. Goldstein, S. Argov, J. Microsc. 215 (2004) 86.

[15] R.K. Sahu, S. Argov, E. Bernshtain, A. Salman, S. Walfisch, J. Goldstein, S. Mordechai, Scand. J. Gastroenterol. 39 (2004) 557.

[16] M.J. Walsh, M.N. Singh, H.M. Pollock, L.J. Cooper, M.J. German, H.F. Stringfellow, N.J. Fullwood, E. Paraskevaidis, P.L. Martin-Hirsch, F.L. Martin, Biochem. Biophys. Res. Commun. 352 ( 2007) 213

[17] F.L. Martin, N.J. Fullwood NJ, Proc. Natl. Acad. Sci. U S A. 104 (2007) E1.

[18] A. Hammiche, L. Bozec, M. Conroy, H.M. Pollock, G. Mills, J.M.R. Weaver, D.M. Price, M. Reading, D.J. Hourston, M. Song, J. Vac. Sci. Tech. B 18 (2000) 1322.

[19] L.P. Choo, D.L. Wetzel, W.C. Halliday, M. Jackson, S.M. LeVine H.H. Mantsch, Biophys. J. 71 (1996) 1672. 
[20] A. Hammiche, L. Bozec, M.J. German, J.M. Chalmers, N.J. Everall, G. Poulter, M. Reading, D.B. Grandy, F.L. Martin, H.M. Pollock, Spectroscopy 19 (2004) 20.

[21] N. Efron, I. Perez-Gomez, H.A. Mutalib, J. Hollingsworth, Cont. Lens Anterior Eye 24 (2001) 16.

[22] F. L. Martin, M. J. German, E. Wit, T. Fearn, N. Ragavan, H. M.

Pollock, J. Computat. Biol. 14 (2007) 1176.

[23] D. Ami D, T. Neri, A. Natalello, P. Mereghetti, S.M. Doglia, M. Zanoni, M. Zuccotti, S. Garagna, C.A. Redi, Biochim. Biophys. Acta. (2008) 98.

[24] T.D. Wang, G. Triadafilopoulos, J.M. Crawford, L.R. Dixon, T. Bhandari, P. Sahbaie, S. Friedland, R. Soetikno, C.H. Contag, Proc. Natl. Acad. Sci. U. S. A. 104 (2007).

[25] M.C Chang, J.Tanaka, Biomaterials 23 (2002) 4811.

[26] A. Polyanichko, H. Wieser, Biopolymers 78 (2005) 329.

[27] F. Keilmann, Vib. Spectrosc. 29 (2002) 109.

[28] A. Hammiche, M.J. German, R. Hewitt, H.M. Pollock, F.L. Martin, Biophys. J. 88 (2005) 3699.

[29] M. Jackson, H.H. Mantsch, Crit. Rev. Biochem. Mol. 30 (1995) 95.

[30] S. Boydston-White, T. Gopen, S. Houser, J. Bargonetti, M. Diem, Biospectroscopy 5 (1999) 219.

[31] O.M. Kvalheim, in J.M. Chalmers, P.R. Griffiths, Handbook of Vibrational Spectroscopy 3, (2002) 2079.

[32] N.J. Fullwood, Nat. Chem. Biol. 3 (2007) 435.

[33] C.S. Potten, M. Loeffler, Development 110 (1990) 1001.

[34] A. Pajoohesh-Ganji, M.A. Stepp, Biol. Cell. 97 (2005) 265.

[35] O. Grude, A. Hammiche, H.M. Pollock, A.J. Bentley, M.J. Walsh, F.L. Martin, N.J. Fullwood, J.Microsc. 228 (2007) 366.

[36] K. Georgopoulos, Nat. Rev. Immunol. 2 (2002) 162.

[37] H. Tagoh, S. Melnik, P. Lefevre, S. Chong, A.D. Riggs, C. Bonifer, Blood 103 (2004) 2950. 